\title{
A FAMILY OF GRADED MODULES ASSOCIATED TO A MODULE
}

\author{
FUTOSHI HAYASAKA
}

This note is a summary of the paper [9] with E. Hyry (University of Tampere). In this note we introduce a certain family of graded modules associated to a given module. These modules provide a natural extension of the notion of the associated graded ring of an ideal. We will investigate their properties. In particular, we will try to extend the Rees theorem on the associated graded ring of an ideal generated by a regular sequence to this context.

\section{INTRODUCTION}

Let $A$ be a commutative ring and let $J$ be an ideal in $A$. In 1957, Rees proved in [19] that the associated graded ring

$$
G(J)=A / J \oplus J / J^{2} \oplus J^{2} / J^{3} \oplus \cdots
$$

of $J$ is isomorphic to the polynomial ring over $A / J$, if the ideal $J$ is generated by a regular sequence on $A$. In particular, the module $J^{\ell} / J^{\ell+1}$ is $A / J$-free for all $\ell \geq 0$. Rees's theorem is a key result for the applications of the associated graded ring in commutative algebra and algebraic geometry.

Recently many authors have investigated graded structures associated to modules, especially in connection with the theory of Buchsbaum-Rim multiplicities. Several results valid in the ideal case have been extended to the module case (for example, see $[2,12,13,15,17,18,20,21]$ ). However, a good notion of an associated graded ring of a module satisfying a suitable version of Rees's theorem seems to be lacking.

Two possible candidates for the associated graded ring of a module appear in the article [12] of Katz and Kodiyalam. Let $A$ be a Noetherian ring and let $F$ be a free $A$-module of rank $r>0$. Let $M$ be a submodule of $F$ and let $R=\mathcal{R}(M)$ be the Rees algebra of $M$, which is the subalgebra of the polynomial $\operatorname{ring} S=\operatorname{Sym}_{A}(F)$ defined as the image of the natural 
homomorphism $\operatorname{Sym}_{A}(M) \rightarrow \operatorname{Sym}_{A}(F)$. Let $I(M)$ be the 0-th Fitting ideal $\operatorname{Fitt}_{0}(F / M)$ of $F / M$. Katz and Kodiyalam investigated the graded $A / I(M)$-algebra

$$
R / I(M) R=A / I(M) \oplus M / I(M) M \oplus M^{2} / I(M) M^{2} \oplus \cdots,
$$

where $M^{\ell}$ denotes the homogeneous component $R_{\ell}$ of degree $\ell$ in $R$. When $r=1$, the ring $R / I(M) R$ is exactly the associated graded ring of the ideal $M$ in $A$. In order to study the Buchsbaum-Rim polynomial, they also introduced a graded $R / I(M) R$-module, namely

$$
R F / R^{+}=F / M \oplus M F / M^{2} \oplus M^{2} F / M^{3} \oplus \cdots .
$$

When $r=1$, this module coincides with the ordinary associated graded ring of the ideal $M$ in $A$. They observe in the proof of [12, Proposition 3.4] that the module $M^{\ell} F / M^{\ell+1}$ is a direct sum of $\left(\begin{array}{c}\ell+r \\ r\end{array}\right)$-copies of $F / M$, if $A$ is a two dimensional regular local ring and $M$ is a parameter module in $F$ (i.e., the length of $F / M$ is finite and the number of generators of $M$ is just $r+1)$. This can be viewed as the module version of Rees' theorem. The goal of this note is to generalize this observation as follows:

Theorem 1.1. Let $A$ be a Noetherian ring and let $F$ be a free A-module of rank $r>0$. Let $M$ be a submodule of $F$ such that $\widetilde{M}$ is a perfect matrix of size $r \times(r+1)$. Then the natural surjective homomorphism

$$
(F / M)\left[Y_{1}, \ldots, Y_{r+1}\right] \longrightarrow R F / R^{+}
$$

of $R / I(M) R$-modules is an isomorphism. In particular, the A-module $M^{\ell} F / M^{\ell+1}$ is a direct sum of $\left(\begin{array}{c}\ell+r \\ r\end{array}\right)$-copies of $F / M$ for all $\ell \geq 0$.

Here $\widetilde{M}$ denotes the matrix whose columns correspond to the generators of $M$ with respect to a fixed basis of $F$. Moreover, we say that the matrix $\widetilde{M}$ perfect if $I(M)$ is a proper ideal having the maximal possible grade.

As a corollary, we have the following.

Corollary 1.2 (cf. [12]). Let $(A, \mathfrak{m})$ be a Cohen-Macaulay local ring of dimension two. Let $F=A^{r}$ be a free module of rank $r>0$ and $M$ a parameter module in $F$. Then the map

$$
\varphi_{1}:(F / M)\left[Y_{1}, \ldots, Y_{r+1}\right] \longrightarrow R F / R^{+}
$$

is an isomorphism and hence the module $M^{\ell} F / M^{\ell+1}$ is a direct sum of $\left(\begin{array}{c}\ell+r \\ r\end{array}\right)$-copies of $F / M$ for all $\ell \geq 0$. 
This is a direct consequence of Theorem 1.1. Since $A$ is a two-dimensional Cohen-Macaulay local ring, the matrix $\widetilde{M}$ of a parameter module $M$ is perfect of size $r \times(r+1)$. Thus the assertion follows from Theorem 1.1.

In section 2, inspired by the article of Katz and Kodiyalam, we are going to introduce a family of graded $R / I(M) R$-modules $\left\{G_{t}(M)\right\}_{t \geq 0}$, where

$$
G_{t}(M)=S_{t} / M S_{t-1} \oplus M S_{t} / M^{2} S_{t-1} \oplus M^{2} S_{t} / M^{3} S_{t-1} \oplus \cdots .
$$

This includes the above two graded modules $R / I(M) R=G_{0}(M)$ and $R F / R^{+}=G_{1}(M)$. We can then ask when the natural surjective homomorphisms $\varphi_{t}:\left(S_{t} / M S_{t-1}\right)\left[Y_{1}, \ldots, Y_{n}\right] \rightarrow G_{t}(M)$ are isomorphisms. In section 3, we will discuss the generic case. It turns out that in the generic case the maps $\varphi_{t}$ are always isomorphisms (see Proposition 3.1). We can then show that the general case can be reduced to this case provided that a certain condition $\mathcal{P}_{t}$ holds in the generic case (see Theorem 3.2). Finally, in section 4 , we will prove in Theorem 4.1 that the condition $\mathcal{P}_{1}$ holds true in the case of a generic $r \times(r+1)$ matrix. This will imply our main Theorem 1.1.

\section{The ASSOciated GRADED MODUles of A MODUle}

Let $A$ be a Noetherian ring and let $F$ be a free $A$-module with a basis $\left\{t_{1}, \ldots, t_{r}\right\}$. Let $M$ be a submodule of $F$ with generators $c_{1}, \ldots, c_{n}$ and the matrix $\widetilde{M}=\left(c_{i j}\right)$. We put $I(M)=I_{r}(\widetilde{M})=\operatorname{Fitt}_{0}(F / M)$. Let $S=A\left[t_{1}, \ldots, t_{r}\right]$ be the symmetric algebra of $F$. Let $R=\mathcal{R}(M)$ be the Rees algebra of $M$, which is now the $A$-subalgebra of $S$ generated by $c_{1}, \ldots, c_{n}$. For each integer $\ell \geq 0$, we denote by $M^{\ell}$ the homogeneous component $R_{\ell}$ of degree $\ell$ in $R$. We always understand products and powers of modules to be taken inside the symmetric algebra $S$ of our fixed free module $F$. We put $R^{+}=\oplus_{\ell>0} M^{\ell}$.

Let $\mathcal{R}(M S)$ be the Rees algebra of the ideal $M S$ in $S$, which is the $S$ subalgebra $S\left[c_{1} T, \ldots, c_{n} T\right]$ of the polynomial ring $S[T]$. The $\operatorname{ring} \mathcal{R}(M S)$ becomes a bi-graded $A$-algebra by letting $\operatorname{deg} t_{i}=(0,1), \operatorname{deg} c_{j} T=(1,0)$. That is,

$$
\mathcal{R}(M S) \cong \bigoplus_{p, q \geq 0} M^{p} S_{q}
$$


Now consider the bi-graded $A / I(M)$-algebra

$$
\mathcal{G}=\mathcal{G}(M S) \otimes_{A}(A / I(M))
$$

where $\mathcal{G}(M S)=\mathcal{R}(M S) /(M S) \mathcal{R}(M S)$ is the associated graded ring of $M S$. So

$$
\mathcal{G} \cong \bigoplus_{p, q \geq 0} M^{p} S_{q} / M^{p+1} S_{q-1}
$$

Here we set $M S_{-1}=I(M)$.

Definition 2.1. For any non-negative integer $t \geq 0$, we set

$$
G_{t}(M)=\bigoplus_{p \geq 0} \mathcal{G}_{(p, t)}=R S_{t} / R^{+} S_{t-1},
$$

and call the module $G_{t}(M)$ as the associated graded module of $M$ of type $t$

Let $S\left[Y_{1}, \ldots, Y_{n}\right]$ be a polynomial ring over $S$ with $\operatorname{deg} Y_{j}=(1,0)$. Consider the bi-graded homomorphism

$$
\phi:(S / M S)\left[Y_{1}, \ldots, Y_{n}\right] \longrightarrow \mathcal{G} ; Y_{j} \mapsto c_{j}+(M S)^{2} .
$$

Taking the degree $(*, t)$-part, we obtain the homomorphism

$$
\varphi_{t}:\left(S_{t} / M S_{t-1}\right)\left[Y_{1}, \ldots, Y_{n}\right] \longrightarrow G_{t}(M),
$$

of graded $(A / I(M))\left[Y_{1}, \ldots, Y_{n}\right]$-modules. With this notation, our problem is now the following.

Problem 2.2. Let $M$ be a submodule of $F$. Assume that the matrix $\widetilde{M}$ is perfect of size $r \times n$ (i.e., $I(M)$ is a proper ideal and grade $I(M)=$ $n-r+1)$. Is the map $\varphi_{t}$ then an isomorphism?

We note here that modules with a perfect matrix are called complete intersection modules in [21]. This problem can be reduced to the generic case provided that a certain condition $\mathcal{P}_{t}$ holds in the generic case.

\section{THE REDUCTION TO THE GENERIC CASE}

In this section we will reduce Problem 2.2 to the generic case. Let $X=\left(X_{i j}\right)$ be a generic matrix of size $r \times n$ and let

$$
B=\mathbb{Z}[X]=\mathbb{Z}\left[X_{i j} \mid 1 \leq i \leq r, 1 \leq j \leq n\right]
$$


be the corresponding polynomial ring over the ring of integers $\mathbb{Z}$. Let $G=B^{r}$ be a free module of rank $r$ and let $N \subseteq G$ be a submodule of $G$ such that $\widetilde{N}$ is the generic matrix $X$. Let $V=\operatorname{Sym}_{B}(G)=B\left[t_{1}, \ldots, t_{r}\right]$ be the polynomial ring over $B$ and let $U=\mathcal{R}(N)$ be the Rees algebra of $N$. Let

$$
x_{j}=X_{1 j} t_{1}+\cdots+X_{r j} t_{r} \in V, \quad(j=1, \ldots, n)
$$

be the generators of $N$. For a generic matrix of an arbitrary size, one can check that the sequence $x_{1}, \ldots, x_{n}$ form a $d$-sequence on $V$ and hence the ideal $N V$ is of linear type ([22]). Furthermore, in the generic case, we have the following.

Proposition 3.1. For any integer $t \geq 0$, the map

$$
\varphi_{t}:\left(V_{t} / N V_{t-1}\right)\left[Y_{1}, \ldots, Y_{n}\right] \longrightarrow G_{t}(N)=U V_{t} / U^{+} V_{t-1}
$$

is an isomorphism. In particular, the natural surjective homomorphism

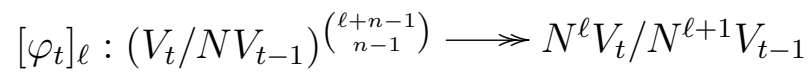

is an isomorphism of $B$-modules for all $\ell \geq 0$.

We now consider the following condition $\mathcal{P}_{t}$ : The $B$-module $V_{\ell+t} / N^{\ell} V_{t}$ is perfect of grade $n-r+1$ for all $\ell>0$.

We will see in the next Theorem 3.2 that if this condition holds true, then the general case of our Problem 2.2 can be reduced to the generic case. For this, we recall here that $A$ is a Noetherian ring, $F$ is a free $A$-module of rank $r>0$ and $S$ is the polynomial ring $A\left[t_{1}, \ldots, t_{r}\right]$.

Theorem 3.2. Let $M$ be a submodule of $F$ with a perfect matrix $\widetilde{M}$ of size $r \times n$. Let $0 \leq t(\leq n-r)$ be a fixed integer. If condition $\mathcal{P}_{t}$ holds true, then the map

$$
\varphi_{t}:\left(S_{t} / M S_{t-1}\right)\left[Y_{1}, \ldots, Y_{n}\right] \longrightarrow G_{t}(M)
$$

is an isomorphism.

Here we give some remarks on condition $\mathcal{P}_{t}$.

Remark 3.3. We do not know whether condition $\mathcal{P}_{t}$ holds true or not, except for the following cases. 
(1) The free resolution of $V_{t+1} / N V_{t}$ is given by the generalized Koszul complex (see $[4,14]$ and also [8, Appendix A2.6]). So the length of this resolution is just $n-r+1$ when $0 \leq t \leq n-r$. However, when $t \geq n-r+1$, the resolution is at least of length $n-r+2$. Hence condition $\mathcal{P}_{t}$ does not hold when $t \geq n-r+1$.

(2) When $t=0$, it is known that condition $\mathcal{P}_{0}$ holds true. This follows from the theorem of Buchsbaum-Eisenbud [5, Corollary 3.2] (see also [13, Proposition 3.3]). Hence $\varphi_{0}$ is an isomorphism if the matrix is perfect (cf. [13, Lemma 3.2]).

(3) When $n=r+1$, condition $\mathcal{P}_{1}$ holds true. This will be proved in the next section (Theorem 4.1).

\section{A Sketch of Proof of Theorem 1.1}

In this final section we will give a sketch of proof of Theorem 1.1. Let $X=\left(X_{i j}\right)$ be a generic matrix of size $r \times n$ and let

$$
B=K[X]=K\left[X_{i j} \mid 1 \leq i \leq r, 1 \leq j \leq n\right]
$$

be the corresponding polynomial ring over an arbitrary commutative Noetherian ring $K$. Let $G=B^{r}$ be a free module of rank $r$ and let $N \subseteq G$ be a submodule of $G$ such that $\widetilde{N}$ is the generic matrix $X$. Identify $\operatorname{Sym}_{B}(G)$ with the polynomial ring $V=B\left[t_{1}, \ldots, t_{r}\right]$ and let $U=\mathcal{R}(N)$ be the Rees algebra of $N$. By Theorem 3.2, it is enough to show that condition $\mathcal{P}_{1}$ (stated before Theorem 3.2) holds true when $n=r+1$.

Theorem 4.1. Suppose that $n=r+1$. Then the module $V_{\ell+1} / N^{\ell} V_{1}$ is a perfect $B$-module of grade 2 for all $\ell>0$. In particular, condition $\mathcal{P}_{1}$ (stated before Theorem 3.2) holds true.

Let me give a sketch of proof of Theorem 4.1. We assume in the following that $n=r+1$. We put $x_{j}=X_{1 j} t_{1}+\cdots+X_{r j} t_{r}$ and set $I=$ $\left(x_{1}, \ldots, x_{r+1}\right) V=N V$. Let $\mathcal{R}=\mathcal{R}(I)$ be the Rees algebra of $I$, which is the $V$-subalgebra $V\left[x_{1} T, \ldots, x_{r+1} T\right]$ of the polynomial ring $V[T]$. We regard the ring $V[T]$ as a bi-graded $B$-algebra by letting $\operatorname{deg} t_{i}=(0,1)$, $\operatorname{deg} T=(1,-1)$. Then the Rees algebra $\mathcal{R}$ becomes a bi-graded $B$ subalgebra of $V[T]$. Note that the $B$-module $N^{\ell} V_{t}$ is now isomorphic to the homogeneous component $\mathcal{R}_{\ell, t}$ of $\mathcal{R}$ for all $\ell, t \geq 0$. We thus want to 
show the following:

$$
\operatorname{pd}_{B} \mathcal{R}_{p, 1} \leq 1 \text { for all } p>0 \text {. }
$$

Let $\mathcal{S}=V\left[Y_{1}, \ldots, Y_{r+1}\right]$ be a polynomial ring over $V$. We regard $\mathcal{S}$ as a bi-graded $B$-algebra by setting $\operatorname{deg} Y_{j}=(1,0)$ for all $j=1, \ldots, r+1$. We now have the bi-graded presentation $\varepsilon: \mathcal{S} \rightarrow \mathcal{R} ; Y_{j} \mapsto x_{j} T$ of $\mathcal{R}$. To show the inequality (1), we will first construct a graded $\mathcal{S}$-free resolution of $\mathcal{R}$. This will be achieved by using the $\mathcal{Z}$-complex, which was introduced by Herzog-Simis-Vasconcelos in [11]. We refer the reader to [10, section 3] for a similar approach.

Let $K_{\bullet}(\underline{x} ; \mathcal{S})$ and $K_{\bullet}(\underline{Y} ; \mathcal{S})$ be the Koszul complexes associated to sequences $\underline{x}=x_{1}, \ldots, x_{r+1}$ and $\underline{Y}=Y_{1}, \ldots, Y_{r+1}$ in $\mathcal{S}$, respectively. We denote by $d_{x}$ and $d_{Y}$ the corresponding differentials. These complexes become bi-graded complexes:

$$
\begin{array}{llllllll}
K_{\bullet}(\underline{x} ; \mathcal{S}): & \cdots & \stackrel{d_{x}}{\rightarrow} & K_{i+1}(0,-i-1) & \stackrel{d_{x}}{\rightarrow} & K_{i}(0,-i) & \stackrel{d_{x}}{\rightarrow} & \cdots \\
K_{\bullet}(\underline{Y} ; \mathcal{S}): & \cdots & \stackrel{d_{Y}}{\rightarrow} & K_{i+1}(-i-1,0) & \stackrel{d_{Y}}{\rightarrow} & K_{i}(-i, 0) & \stackrel{d_{Y}}{\rightarrow} & \cdots,
\end{array}
$$

where $K_{i}=\wedge^{i} \mathcal{S}^{r+1}$. Let

$$
\mathcal{Z}_{i}=\operatorname{Ker}\left(K_{i}(0,-i) \stackrel{d_{x}}{\rightarrow} K_{i-1}(0,-i+1)\right)
$$

be the $i$-th module of cycles of $K_{\bullet}(\underline{x} ; \mathcal{S})$, which is a graded submodule of $K_{i}(0,-i)$. Since $d_{x} \circ d_{Y}+d_{Y} \circ d_{x}=0,\left\{\mathcal{Z}_{\bullet}, d_{Y}\right\}$ is a subcomplex of $K_{\bullet}(\underline{Y} ; \mathcal{S})$. This complex is called the $\mathcal{Z}$-complex associated to a sequence $\underline{x}$ and denoted by $\mathcal{Z}_{\bullet}(\underline{x})([11])$. Note that the 0 -th homology $H_{0}\left(\mathcal{Z}_{\bullet}(\underline{x})\right) \cong$ $\operatorname{Sym}_{V}(I)$. Since $\underline{x}$ is a $d$-sequence on $V, \mathcal{Z}_{\bullet}(\underline{x})$ is acyclic with the 0 -th homology $H_{0}\left(\mathcal{Z}_{\bullet}(\underline{x})\right) \cong \mathcal{R}([11$, Theorem 5.4]). Hence we have the graded exact sequence

$0 \rightarrow \mathcal{Z}_{n-1}(-n+1, n-1) \stackrel{d_{Y}}{\rightarrow} \cdots \stackrel{d_{Y}}{\rightarrow} \mathcal{Z}_{2}(-2,2) \stackrel{d_{Y}}{\rightarrow} \mathcal{Z}_{1}(-1,1) \stackrel{d_{Y}}{\rightarrow} \mathcal{S} \stackrel{\varepsilon}{\rightarrow} \mathcal{R} \rightarrow 0$.

If we now resolve each of the modules $\mathcal{Z}_{i}(-i, i)$ by a certain (graded) complex $P_{\bullet}$ and lift the differentials $\mathcal{Z}_{i}(-i, i) \stackrel{d_{Y}}{\rightarrow} \mathcal{Z}_{i-1}(-i+1, i-1)$ to maps $P_{\bullet} i P_{\bullet i-1}$ of complexes, then the associated double complex will give us an $\mathcal{S}$-free resolution of $\mathcal{R}$.

It is well-known that $x_{1}, \ldots, x_{r}$ is a regular sequence on $V$ (see $[1$, Proposition 1]). Hence we have for all $i \geq 2$ the exact sequence

$$
0 \rightarrow K_{r+1}(-i, i-r-1) \stackrel{d_{x}}{\rightarrow} \cdots \stackrel{d_{x}}{\rightarrow} K_{i+1}(-i,-1) \stackrel{d_{x}}{\rightarrow} \mathcal{Z}_{i}(-i, i) \rightarrow 0 .
$$


This gives us a graded $\mathcal{S}$-free resolution $P_{\bullet}$ of $\mathcal{Z}_{i}(-i, i)$ for $i \geq 2$.

In order to find a resolution of $\mathcal{Z}_{1}(-1,1)$, we need to introduce some more notation. Let $K_{\bullet}(\underline{t} ; \mathcal{S})$ be the Koszul complex of the sequence $\underline{t}=t_{1}, \ldots, t_{r}$ in $S$ with the differential $d_{t}$. We interpret it as bi-graded complex

$$
K_{\bullet}(\underline{t} ; \mathcal{S}): \quad \cdots \quad \stackrel{d_{t}}{\rightarrow} L_{i+1}(0,-i-1) \stackrel{d_{t}}{\rightarrow} L_{i}(0,-i) \stackrel{d_{t}}{\rightarrow} \quad \cdots,
$$

where $L_{i}=\wedge^{i} \mathcal{S}^{r}$. Since $\left[t_{1} \cdots t_{r}\right] \circ X=\left[x_{1} \cdots x_{r+1}\right]$, there is a map of complexes $\wedge X: K_{\bullet}(\underline{x} ; \mathcal{S}) \longrightarrow K_{\bullet}(\underline{t} ; \mathcal{S})$. Taking the $\mathcal{S}$-duals, this gives a map $\wedge^{t} X: K^{\bullet}(\underline{t} ; \mathcal{S}) \longrightarrow K^{\bullet}(\underline{x} ; \mathcal{S})$ of complexes, where ${ }^{t} X$ denotes the transpose of $X$. Identifying the Koszul complexes with their duals, we obtain a map of complexes

$$
f_{\bullet}: K_{\bullet}(\underline{t} ; \mathcal{S})(0,-1) \rightarrow K_{\bullet}(\underline{x} ; \mathcal{S})[1],
$$

where each $f_{i}: L_{i}(0,-i-1) \rightarrow K_{i+1}(0,-i-1)$ is a graded homomorphism of degree zero. More explicitly, setting $\Delta_{j}=(-1)^{j-1} \operatorname{det} X_{j}$ where $X_{j}$ is the matrix obtained by deleting $j$-th column of $X$, one can check, for example, that $f_{0}={ }^{t}\left[\Delta_{1} \Delta_{2} \cdots \Delta_{r+1}\right]$ and $f_{r}$ is the identity map.

We then have the following.

Proposition 4.2. With the notation above, there is the following graded resolution of $\mathcal{Z}_{1}(-1,1)$ :

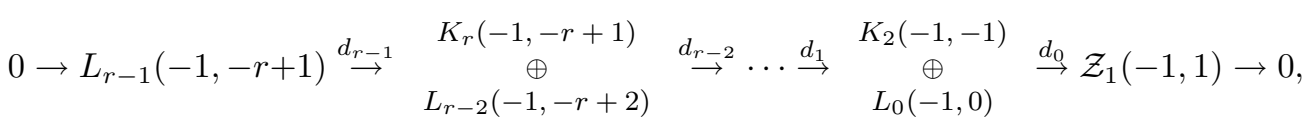

where $d_{0}=\left(\begin{array}{ll}d_{x} & f_{0}\end{array}\right), d_{r-1}=\left(\begin{array}{c}f_{r-1} \\ -d_{t}\end{array}\right)$, and $d_{i}=\left(\begin{array}{cc}d_{x} & f_{i} \\ 0 & -d_{t}\end{array}\right)$ for all $1 \leq i \leq r-2$.

Let $P_{\bullet}$ be the above graded resolution of $\mathcal{Z}_{1}(-1,1)$. Look at the following commutative diagram.

$$
\begin{aligned}
& \begin{array}{lll}
0 & 0 & 0
\end{array}
\end{aligned}
$$

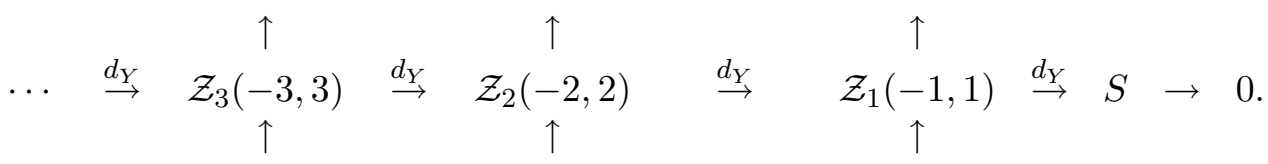

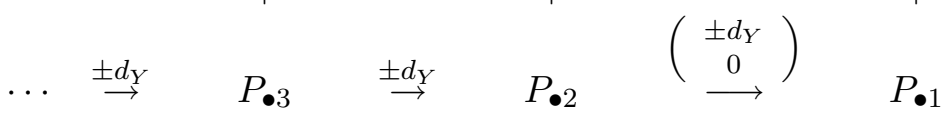


Let $\operatorname{Tot}\left(P_{\bullet \bullet}\right)$ be the total complex of the resulting double complex $P_{\bullet \bullet}$. Now consider the corresponding spectral sequence. Since $\mathcal{Z}_{\bullet}(\underline{x})$ is acyclic with $H_{0}\left(\mathcal{Z}_{\bullet}(\underline{x})\right) \cong \mathcal{R}$, standard arguments yield the following graded $\mathcal{S}$ free resolution of $\mathcal{R}$ :

$$
\operatorname{Tot}\left(P_{\bullet \bullet}\right) \rightarrow \mathcal{S} \rightarrow \mathcal{R} \rightarrow 0 .
$$

Note that this resolution gives us the defining equations of $\mathcal{R}$ :

$$
\mathcal{K}=I_{2}\left(\begin{array}{cccc}
x_{1} & x_{2} & \cdots & x_{r+1} \\
Y_{1} & Y_{2} & \cdots & Y_{r+1}
\end{array}\right)+I_{r+1}\left(\begin{array}{ccc}
Y_{1} & \cdots & Y_{r+1} \\
& & \\
& X &
\end{array}\right) .
$$

Hence, by [3, Theorem (3.3)], it suffices to prove the inequality (1) in the case $K=\mathbb{Z} / \mathfrak{p} \mathbb{Z}$ where $\mathfrak{p}$ is a prime. In other words we can assume that $B=K[X]$ where $K$ is a field.

When $r=1$, the assertion is immediate from the above resolution. In the following we therefore assume that $r \geq 2$. Using the above resolution of $\mathcal{R}$, we can compute $\operatorname{Tor}_{i}^{B}(K, \mathcal{R})$ for $i \geq 2$ as follows:

$$
\operatorname{Tor}_{i}^{B}(K, \mathcal{R}) \cong H_{i-1}(\operatorname{Tot}(\bar{P} \bullet \bullet)) \cong \begin{cases}\mathcal{B}_{i}(i,-i) & (2 \leq i \leq r-1) \\ \bar{L}_{r}(-1,-r) & (i=r)\end{cases}
$$

where $\bar{*}=* \otimes_{B} K$ and $\mathcal{B}_{i}=\operatorname{Im}\left(\bar{K}_{i+1}(-i-1,0) \stackrel{d_{Y}}{\rightarrow} \bar{K}_{i}(-i, 0)\right) \subseteq \bar{K}_{i}(-i, 0)$ is the $i$-th module of boundaries of the complex $K_{\bullet}(\underline{Y} ; \overline{\mathcal{S}})$. Hence we have $\operatorname{Tor}_{i}^{B}\left(K, \mathcal{R}_{p, 1}\right)=(0)$ for all $i \geq 2$, because

$$
\begin{cases}{\left[\mathcal{B}_{i}\right]_{p+i, q-i}=(0)} & \text { if } q<i \\ {\left[\bar{L}_{r}\right]_{p-1, q-r}=(0)} & \text { if } q<r .\end{cases}
$$

Consequently, we have $\operatorname{pd}_{B} \mathcal{R}_{p, 1} \leq 1$. This completes the proof of Theorem 4.1 and hence we have Theorem 1.1.

\section{REFERENCES}

[1] L. L. Avramov, Complete intersections and symmetric algebras, J. Algebra 73 (1981), no. 1, 248-263

[2] J. Brennan, B. Ulrich and W. V. Vasconcelos, The Buchsbaum-Rim polynomial of a module, J. Algebra 241 (2001), 379-392

[3] W. Bruns and U. Vetter, Determinantal Rings, Lecture Notes in Math. 1327, Springer-Verlag Berlin Heidelberg, 1988. 
[4] D. Buchsbaum and D. Eisenbud, Remarks on ideals and resolutions, Symposia Mathematica, Vol. XI (Convegno di Algebra Commutativa, INDAM, Rome, 1971), Academic Press, London (1973), 193-204

[5] D. A. Buchsbaum and D. Eisenbud, Generic free resolutions and a family of generically perfect ideals, Adv. in Math. 18 (1975), 245-301

[6] D. A. Buchsbaum and D. S. Rim, A generalized Koszul complex, Bull. Amer. Math. Soc. 69 (1963), 382-385

[7] D. A. Buchsbaum and D. S. Rim, A generalized Koszul complex. II. Depth and multiplicity, Trans. Amer. Math. Soc. 111 (1964), 197-224

[8] D. Eisenbud, Commutative algebra. With a view toward algebraic geometry, Graduate Texts in Mathematics, 150, Springer-Verlag, New York, 1995

[9] F. Hayasaka and E. Hyry, A family of graded modules associated to a module, to appear in Communications in Algebra

[10] J. Herzog and Y. Kamoi, Taylor complexes for Koszul boundaries, Manuscripta Math. 96 (1998), no. 2, 133-147

[11] J. Herzog, A. Simis and W. V. Vasconcelos, Approximation complexes of blowingup rings, J. Algebra 74 (1982), no. 2, 466-493

[12] D. Katz and V. Kodiyalam, Symmetric powers of complete modules over a twodimensional regular local ring, Trans. Amer. Math. Soc. 349 (1997), 747-762

[13] D. Katz and C. Naudé, Prime ideals associated to symmetric powers of a module, Comm. Algebra 23 (1995), no. 12, 4549-4555

[14] D. Kirby, A sequence of complexes associated with a matrix, J. London Math. Soc. 7 (1974), 523-530

[15] D. Kirby, On the Buchsbaum-Rim multiplicity associated with a matrix, J. London Math. Soc. (2) 32 (1985), no. 1, 57-61

[16] D. Kirby, Generalized Koszul complexes and the extension functor, Comm. Algebra 18 (1990), no. 4, 1229-1244

[17] S. Kleiman and A. Thorup, A geometric theory of the Buchsbaum-Rim multiplicity, J. Algebra 167 (1994), no. 1, 168-231

[18] S. Kleiman and A. Thorup, Mixed Buchsbaum-Rim multiplicities, Amer. J. Math. 118 (1996), no. 3, 529-569

[19] D. Rees, The grade of an ideal or module, Proc. Cambridge Philos. Soc. 53 (1957), 28-42

[20] A. Simis, B. Ulrich and W. V. Vasconcelos, Codimension, multiplicity and integral extensions, Math. Proc. Cambridge Philos. Soc. 130 (2001), no. 2, 237-257

[21] A. Simis, B. Ulrich and W. V. Vasconcelos, Rees algebras of modules, Proc. London Math. Soc. 87 (2003), 610-646

[22] I. Swanson and C. Huneke, Integral closure of ideals, rings, and modules, London Mathematical Society Lecture Note Series, 336, Cambridge University Press, Cambridge, 2006

Department of Mathematics, School of Science and Technology, Meiji UNIVERSITY, 214-8571 JAPAN

E-mail address: fhayasaka@math.meiji.ac.jp 